Artículo

\title{
Modelo estadístico para la predicción del rendimiento de grano en maíz
}

Fernando Reyes-González ${ }^{1}$

Arturo Galvis-Spinola ${ }^{1 \S}$

Juan José Almaraz-Suárez ${ }^{1}$

Teresa Marcela Hernández-Mendoza ${ }^{2}$

${ }^{1}$ Postgrado en Edafología-Colegio de Postgraduados-Campus Montecillo. Carretera México-Texcoco km 36.5, Montecillo, Texcoco, Estado de México, México. CP. 56230. (frg_58@hotmail.com; jalmaraz@ colpos.mx). ${ }^{2}$ Departamenteo de Irrigación-Universidad Autónoma Chapingo. Carretera MéxicoTexcoco km 38.5, Chapingo, Texcoco, Estado de México, México. CP. 56230. (teresa_hdz 2004@yahoo.com).

$\S$ Autor para correspondencia: galvispinola@gmail.com.

\section{Resumen}

El crecimiento de la población mundial conlleva a la demanda de alimentos, y estos se deben obtener mediante el uso eficiente de los recursos, esto se podría lograr mediante la planificación y priorización de los factores que intervienen en los procesos de producción. Los modelos de simulación son una herramienta con la que se puede visualizar escenarios y cuantificar los insumos a usar. En el presente trabajo, con datos de los rendimientos máximos de maíz (RG) de 1943 a 2017 obtenidos de experimentos de campo a nivel mundial y con predominancia de datos de Estados Unidos de América (80\%), se generó un modelo estadístico para estimar el rendimiento del grano en maíz $\left(\mathrm{RG}_{\mathrm{E}}\right)$ y sirva de apoyo para la toma de decisiones de quienes participan en el proceso de producción de maíz para grano. Las variables de mayor peso para expresar el modelo fueron: densidad de población $\left(\mathrm{D}_{\mathrm{P}}\right)$, dosis de potasio $(\mathrm{K})$, lamina de riego $\left(\mathrm{L}_{\mathrm{R}}\right)$, dosis de nitrógeno $(\mathrm{N})$ y dosis de fósforo $(\mathrm{P})$ y se usaron para generar el modelo con el método de regresión múltiple stepwise, y se expresó como: $\mathrm{RG}_{\mathrm{E}}=3.158205+0.693319\left(\mathrm{D}_{\mathrm{P}}\right)-0.022246(\mathrm{~K})+0.005990\left(\mathrm{~L}_{\mathrm{R}}\right)+$ $0.010687(\mathrm{~N})+0.013794(\mathrm{P})$, tuvo un $\mathrm{R}^{2}=0.73$ y un error estándar de $0.964 \mathrm{Mg} \mathrm{ha}^{-1}$. La DP fue la variable que explicó en mayor proporción el valor del $\mathrm{RG}_{\mathrm{E}}$, con el análisis de datos de RG se observó el incremento de la tasa de siembra a través del tiempo para lograr una mayor $\mathrm{D}_{\mathrm{P}} \mathrm{e}$ incrementar el RG, lo cual generó la demanda de insumos.

Palabras clave: Zea mays L., densidad de población, nitrógeno.

Recibido: enero de 2021

Aceptado: marzo de 2021 


\section{Introducción}

La tasa más alta de aumento del rendimiento en híbridos de maíz de 1950 a 1999 en el este de Nebraska, para condiciones de secano fue de $0.05 \mathrm{Mg} \mathrm{ha}^{-1} \mathrm{año}^{-1}$ en suelos con alta capacidad de retención de agua, seguida por la de riego con $0.028 \mathrm{Mg} \mathrm{ha}^{-1}$ año $^{-1}$ (Mason et al., 2008). Las tendencias de rendimiento de 1987 a 2015 indicaron un aumento comparable de acuerdo a las latitudes $\left(108,97\right.$ y $117 \mathrm{~kg} \mathrm{ha}^{-1}$ año $^{-1}$ para $35-40^{\circ}, 40-45^{\circ}$ y $45-50^{\circ}$ latitud norte, respectivamente) y se registró una mejora en los entornos de alto (AR) y muy alto rendimiento (MAR) a tasa máxima promedio de $50 \mathrm{~kg} \mathrm{ha}^{-1} \mathrm{año}^{-1}$ (Assefa et al., 2017).

La optimización de los elementos agrotécnicos reduce los efectos climáticos nocivos (Sárvári y Pepó, 2014). La contribución al rendimiento de los factores (resistencia transgénica a insectos, fungicida con estrobilurina, fertilización con fósforo P-azufre S-zinc $\mathrm{Zn}$ y fertilización con nitrógeno N) fue mayor cuando se aplicó como parte de un complemento completo de insumos que cuando se agregó individualmente (Ruffo et al., 2015). La tecnología y el manejo fueron los factores más limitantes, seguidos por la precipitación y el suelo en regiones maiceras de China (Zhao et al., 2018). El reto es desenredar la interacción de los factores que pueden ser manipulados por los agricultores para incrementar el rendimiento (Van Loon et al., 2019).

El rendimiento reducido en la temporada larga de lluvias en 2000 y 2001 se atribuyó a la mala distribución de la precipitación (Shisanya et al., 2009). La precipitación fue el factor limitante para que el potencial de rendimiento limitado por agua alcanzara el nivel del potencial en cuatro regiones maiceras en China (Liu et al., 2017). En maíz de secano mediante correlación, la precipitación estacional explicó al rendimiento de grano en un 66\% (Limón-Ortega et al., 2016).

El efecto de la humedad almacenada en el suelo y disponible para la planta más una fertilidad adecuada y un alto nivel de manejo, indicaron que el potencial para producir maíz está determinado por tales capacidades del suelo (Leeper et al., 1974). La textura del suelo debe ser un criterio importante sobre el cual hacer recomendaciones de la dosis estática de $\mathrm{N}$ para optimizar el rendimiento y evitar la acumulación de nitrato residual en el suelo (Alotaibi et al., 2018).

Persisten problemas a resolver, como precisar la dosis de fertilizante que debe aplicarse para incrementar la eficiencia de los nutrimentos y la rentabilidad de la producción, sin deterioro de los recursos naturales (Bugarín-Montoya et al., 2002) y los relacionados al contenido relativo de los nutrimentos en un cultivo están conectados con problemas relativos a técnicas de fertilización (Alcántar-González et al., 2016). El consumo del N necesario para una tasa de crecimiento máximo depende en parte de la tasa de crecimiento cuando no está limitado por la falta de agua o nutrientes; el porcentaje de $\mathrm{N}$ en cultivos ampliamente diferentes parece estar determinado no tanto por la especie sino por el peso total de materia seca por unidad de área (Greenwood, 1983).

El maíz requiere entre 20 a $25 \mathrm{~kg} \mathrm{~N}^{-1}$ por cada tonelada de grano producida (Melgar y TorresDuggan, 2004). El diseño de un sistema integrado de manejo de cultivos y de $\mathrm{N}$ es una aproximación alternativa para maximizar el uso de la radiación solar y los periodos de temperatura favorable, para lograr un alto rendimiento y una nueva eficiencia en el uso del N (Guo et al., 2016). El efecto residual de la aplicación de $\mathrm{P}$ al voleo en la producción de maíz y caupí (Vigna unguiculata L.), como medición del rendimiento y el P del suelo, fue mayor a altas dosis aplicadas (Smyth y Cravo, 1990). 
La aplicación balanceada de N, P y S produjo un incremento en los rendimientos respecto a los tratamientos con aplicaciones desbalanceadas o sin aplicación, la máxima absorción de nutrientes se relacionó con un mayor rendimiento (Ciampitti et al., 2010). Las concentraciones en la hoja de la mazorca de N-P-S-cobre $(\mathrm{Cu})$ y de Fe explicaron la variación del rendimiento de grano en más del 50\% y 40\%, respectivamente (Kovács y Vyn, 2017). El K representa al catión que es absorbido en mayor cantidad por las plantas, las gramíneas son conocidas como acumuladoras de $\mathrm{K}$ (Alcántar-González et al., 2016). Bajo estrés por sequía, la pulverización de $\mathrm{K}$ y hierro (Fe) podrían reducir tal estrés en maíz (Zare et al., 2014). La aplicación foliar de 1-3\% y 0.1-0.2\% de K y Zn respectivamente, fue más beneficiosa en condiciones limitadas de riego y en etapa vegetativa dio como resultado un mejor crecimiento y un mayor rendimiento (Amanullah et al., 2016).

Cuando el número de plantas por área va más allá del óptimo hay consecuencias perjudiciales para la ontogenia de la mazorca, y resulta en esterilidad (Sangoi, 2000). El aumento de la densidad de las plantas puede mejorar la biomasa y el rendimiento y también aumenta la competencia por los recursos entre plantas, en una alta densidad, los cambios en la estructura y las funciones de la planta individual y poblacional afectan la morfogénesis de estas, la acumulación de carbohidratos en el tallo, el tejido de la corteza y la resistencia mecánica del tallo, la estructura y funcionamiento de la raíz y son más susceptibles al acame (Jun et al., 2017).

Los agricultores orgánicos pueden mejorar la supresión de malezas en maíz al intercalar con cultivos de cobertura y optimizar el rendimiento al sembrar maíz a una tasa más baja de lo que típicamente se utiliza con cultivo de cobertura (Youngerman et al., 2018).

La disponibilidad de híbridos precoces con menor altura, menor número de hojas, hojas verticales, estilos más pequeños y sincronía entre la floración masculina y femenina ha mejorado la resistencia a altas densidades sin mostrar esterilidad excesiva y ha permitido que intercepte y use la radiación solar de manera más eficiente, contribuyendo al aumento potencial de rendimiento (Sangoi, 2000).

Utilizar la diversidad genética ha sido un pilar de las mejorías de la producción anterior a la secuenciación de ADN de alto rendimiento; las bioinformáticas relacionadas y tecnologías en genética pueden ser empleadas para detectar la variabilidad genética oculta y entender las funciones de los genes (Phillips, 2010). Es importante el cultivo de nuevos materiales genéticos para aumentar el potencial de rendimiento y satisfacer la creciente demanda de alimentos (Tao et al., 2015).

Un modelo puede ser una representación conceptual, numérica o gráfica de un objeto, sistema, proceso, actividad o pensamiento; destaca las características que el modelador considera importantes del fenómeno en cuestión (García, 2008), puede integrar variables climáticas, edáficas y genéticas al diagnóstico de la fertilización N de los cultivos (García, 2005); la información generada es base para mejorar la sincronización de aplicaciones de pesticidas, fertilización, riego y la cosecha (Verdugo-Vásquez et al., 2016). El modelo APSIM-Maize calibrado en la llanura del norte de China (PNC) explicó > 63\% las variaciones en el rendimiento de maíz (Wang et al., 2014).

La brecha de rendimiento determinada con el modelo MCWLA-Maize disminuyó aproximadamente $2 \%$ por año en las principales regiones maiceras en China (Tao et al., 2015). En diferentes ubicaciones e independientemente del método de reporte, los datos de la estimación en V7 predijeron el rendimiento $\left(\mathrm{R}^{2}>0.7\right)$ y se pueden usar para predecir los rendimientos en condiciones sin sequía en Nueva York (Tagarakis y Ketterings, 2017). 
El objetivo de este trabajo fue discernir entre diferentes condiciones ambientales y de manejo, aquellas variables que más influyan sobre el RG. Con base en ello, crear un modelo matemático para determinar el $\mathrm{RG}_{\mathrm{E}}$, aplicable en diferentes condiciones de producción.

Las hipótesis de trabajo fueron: el RG es función de las condiciones ambientales y de manejo agronómico, las variables de clima influyen de manera más significativa sobre el RG, en comparación con las que componen el manejo agronómico y el RG es consecuencia de la incidencia de múltiples variables, razón por la cual, el modelo de regresión múltiple permite determinar de manera más apropiada el $\mathrm{RG}_{\mathrm{E}}$ para distintas condiciones ambientales y de manejo.

\section{Materiales y métodos}

La base de datos de los rendimientos máximos de grano de maíz (RG) se generó con información buscada en el año 2019 en las bases: Science Direct y ASCESS DL con las palabras clave 'high yield' 'corn' 'maize' del periodo de 1943 a 1946 y de 1948 a 2017, se seleccionaron alrededor de diez valores de RG representativos por año, estudios que abarcaron más de un año de investigación representaron los RG de más de un año. Dentro de los RG registrados en la literatura mediante ensayos no se consideró a alguno de México debido a que hubo registros superiores en el resto del mundo.

De cada estudio se capturó la localización, año(s) estudiado(s), material genético, manejo agronómico, propiedades y características del suelo; condiciones climáticas y la respuesta de la experimentación. El RG se ajustó a $13.5 \%$ de humedad de grano y se expresó en $\mathrm{Mg} \mathrm{ha}^{-1}$. Por la diversidad de los ensayos, las variables que reportaron no siempre fueron las mismas. La clasificación de variables fue: de estudio, otras variables reportadas, y del sitio. La variable dependiente estudiada fue el RG, como el principal producto de maíz, y el cual depende de diversos factores.

El análisis de datos fue: identificación y clasificación de las variables independientes de cada experimento; tendencia general de los RG; a través, de los años mediante regresión lineal (RL), tendencia de los RG a través de los años por el régimen hídrico reportado, mediante RL; tendencia de los RG a través de los años para cada variable independiente identificada indistintamente del régimen hídrico reportado, mediante $\mathrm{RL}$, tendencia de los RG; a través, de los años para cada variable independiente identificada, de acuerdo al régimen hídrico reportado, mediante RL, filtración de las variables independientes para la creación del modelo en el orden siguiente: lámina de riego, dosis de $\mathrm{N}$, dosis de $\mathrm{P}$, dosis de $\mathrm{K}$ y densidad de población, selección y ordenamiento de las variables independientes para su análisis mediante el método de regresión múltiple stepwise, generación de las ecuaciones: general, por régimen hídrico y de acuerdo a la textura del suelo, por sistema de labranza y por sistema de riego y validación del modelo: RG observado $v s$ RG estimado mediante RL.

La metodología de análisis de los factores fue realizada mediante un programa de cálculo 'stepwise', que incluye o elimina variables predictoras según la incidencia que esto provoca en el estadístico empleado para valorar la significancia de la relación (De la Casa, 1992), los análisis estadísticos se realizaron con el programa R (R Core Team, 2017). 


\section{Resultados y discusión}

\section{Distribución espacial y temporal de los datos}

Los estudios analizados representaron ochenta distintas ubicaciones y $80 \%$ se localizaron en los Estados Unidos de América (Cuadro 1), el resto se localizó en Rumania, Líbano, Canadá, España, Eslovenia, Francia, China y Argentina, se alcanzó una representatividad de distintos entornos de producción y esquemas de manejo. A través, de los años se incrementó la densidad de la población, el uso de fertilizantes y de variedades mejoradas en los sistemas de producción, con el fin de lograr los más altos rendimientos. Las investigaciones futuras podrían continuar centrándose en la mejora del rendimiento y la reducción de los entornos de rendimiento bajo y rendimiento medio con tecnologías mejoradas del manejo de cultivo y uso de híbridos (Assefa et al., 2017).

Cuadro 1. Resumen de los datos de rendimiento de grano (RG) del periodo (1943-2017).

\begin{tabular}{cccc}
\hline \multirow{2}{*}{ Parámetros } & General & \multicolumn{2}{c}{ Régimen hídrico } \\
\cline { 3 - 4 } & & Riego & Secano \\
\hline Observaciones & 732 & 331 & 23 \\
Mínimo $\left(\mathrm{Mg} \mathrm{ha}^{-1}\right)$ & 1.996 & 5.801 & 5.828 \\
Primer cuartil $\left(\mathrm{Mg} \mathrm{h}^{-1}\right)$ & 8.097 & 10.241 & 9.154 \\
Mediana $\left(\mathrm{Mg} \mathrm{ha}^{-1}\right)$ & 10.796 & 11.701 & 10.79 \\
Media $\left(\mathrm{Mg} \mathrm{ha}^{-1}\right)$ & 10.679 & 12.395 & 10.602 \\
Tercer cuartil $\left(\mathrm{Mg} \mathrm{ha}^{-1}\right)$ & 12.64 & 15.414 & 12.668 \\
Máximo $\left(\mathrm{Mg} \mathrm{ha}^{-1}\right)$ & 19.779 & 19.779 & 13.834 \\
\hline
\end{tabular}

\section{Tendencias del rendimiento de grano de maíz de 1943-2017}

El RG promedió $10.679 \mathrm{Mg} \mathrm{ha}^{-1}$, el valor máximo fue de $19.779 \mathrm{Mg} \mathrm{ha}^{-1}$ en el año 2012 y el mínimo de $1.996 \mathrm{Mg} \mathrm{ha}^{-1}$ y se registró en 1959; a través de los años de estudio los datos mostraron una tendencia de tipo alcista ( $p<0.01, \mathrm{R}^{2}=0.74, \mathrm{n}=732$ ) (Figura 1), lo cual comprobó la mejora constante en los sistemas de producción para lograr un mayor RG. Assefa et al. (2017) encontraron un aumento comparable en el rendimiento promedio de maíz en las latitudes (tasas de 108, 97 y $117 \mathrm{~kg} \mathrm{ha}^{-1}$ año $^{-1}$ para las latitudes 35 a $40^{\circ}, 40$ a $45^{\circ}$ y 45 a $50^{\circ}$ latitud norte, respectivamente).

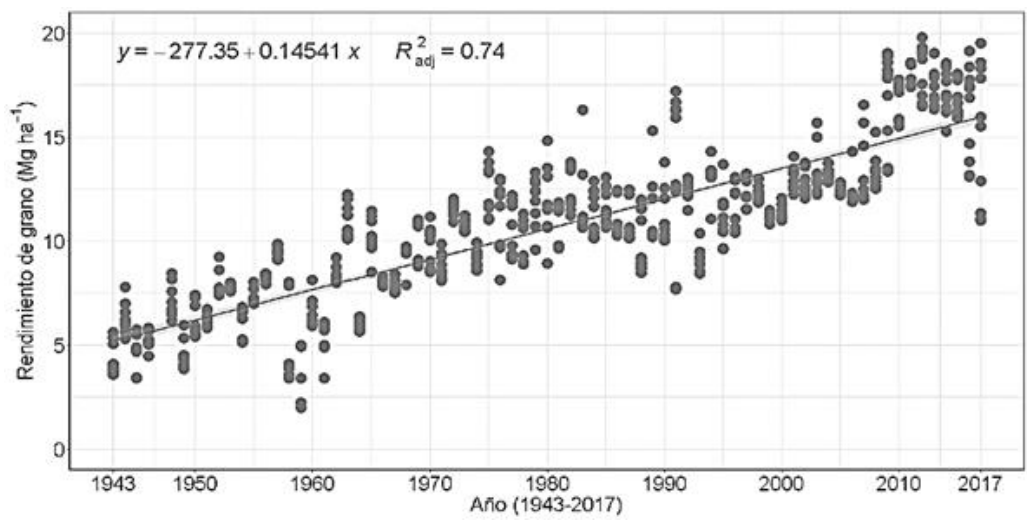

Figura 1. Relación entre los años de estudio y el RG $(p<0.01)$. 


\section{Régimen hídrico}

El RG promedió $12.395 \mathrm{Mg} \mathrm{ha}^{-1}$ y $10.602 \mathrm{Mg} \mathrm{ha}^{-1}$ para la producción de 'riego' y 'secano' respectivamente, mientras que los RG máximos alcanzados fueron de $19779 \mathrm{Mg} \mathrm{ha}^{-1}$ en riego y $13834 \mathrm{Mg} \mathrm{ha}^{-1}$ para secano, en ambos casos de RG y bajo ambos régimenes hídricos de riego ( $p<$ $\left.0.01, \mathrm{R}^{2}=0.7, \mathrm{n}=331\right)$ y secano $\left(p<0.01, \mathrm{R}^{2}=0.59, \mathrm{n}=23\right)$ se observó una tendencia alcista; a través, de los años (Figura 2). La tasa de aumento del rendimiento fue de $0.05 \mathrm{Mg} \mathrm{ha}^{-1} \mathrm{año}^{-1}$ para las condiciones de secano en suelos con alta capacidad de retención de agua y de $0.028 \mathrm{Mg} \mathrm{ha}^{-1}$ año $^{-1}$ para el cultivo bajo riego (Mason et al., 2008). En 2009 los rendimientos de maíz (Cuadro 1) variaron de $9.05 \mathrm{Mg} \mathrm{ha}^{-1}$ para el régimen de secano a $15.5 \mathrm{Mg} \mathrm{ha}^{-1}$ para el riego completo, y en 2010 fueron de $11.7 \mathrm{Mg} \mathrm{ha}^{-1}$ y $15.5 \mathrm{Mg} \mathrm{ha}^{-1}$, respectivamente (Djaman et al., 2013).

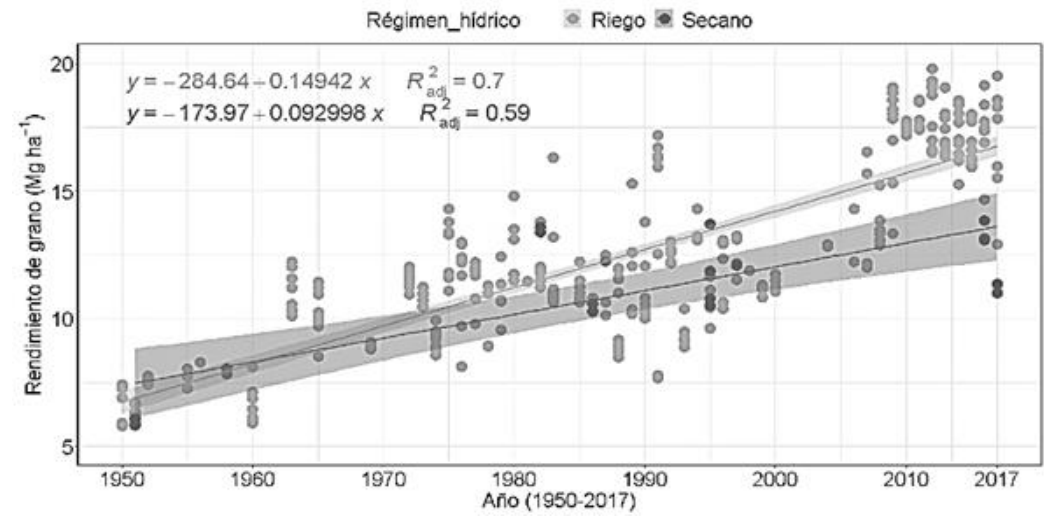

Figura 2. Relación entre los años de estudio y el RG en diferentes regímenes hídricos $(p<0.01)$

\section{Análisis a través del tiempo de las variables del estudio identificadas}

Con el análisis de la dispersión del efecto en RG para cada variable cuantitativa independiente, se delimitaron niveles para poder visualizar la tendencia a través del periodo de estudio (Cuadro 2).

Cuadro 2. Niveles de las variables cuantitativas estudiadas.

\begin{tabular}{cccccccc}
\hline Variable & \multicolumn{7}{c}{ Niveles } \\
\hline $\mathrm{L}_{\mathrm{R}}(\mathrm{mm})$ & $<50$ & $50-224$ & $225-449$ & $450-699$ & $700-900$ & $>900$ & \\
$\mathrm{~N}\left(\mathrm{~kg} \mathrm{ha}^{-1}\right)$ & $<100$ & $100-199$ & $200-299$ & $300-399$ & $400-500$ & $>500$ & \\
$\mathrm{P}\left(\mathrm{kg} \mathrm{ha}^{-1}\right)$ & $<50$ & $50-99$ & $100-149$ & $150-199$ & $200-274$ & $275-325$ & $>325$ \\
$\mathrm{~K}\left(\mathrm{~kg} \mathrm{ha}^{-1}\right)$ & $50-94$ & $95-144$ & $145-199$ & $200-249$ & $250-300$ & $>300$ & \\
$\mathrm{D}_{\mathrm{P}}\left(\right.$ plantas m$\left.^{-2}\right)$ & $<4$ & $4.00-5.49$ & $5.50-7.5$ & $>7.5$ & & & \\
\hline
\end{tabular}

$\mathrm{L}_{\mathrm{R}}=$ lámina de riego; $\mathrm{N}=$ dosis de nitrógeno; $\mathrm{P}=$ dosis de fósforo; $\mathrm{K}=$ dosis de potasio; $\mathrm{D}_{\mathrm{P}}=$ densidad de población.

\section{Lámina de riego}

La respuesta del $\mathrm{RG}$ al riego fue mayor para tasas arriba de $900 \mathrm{~mm}\left(p<0.01, \mathrm{R}^{2}=0.74, \mathrm{n}=18\right) \mathrm{y}$ de 450-699 $\mathrm{mm}\left(p<0.01, \mathrm{R}^{2}=0.73, \mathrm{n}=26\right)$. En cuatro regímenes de riego, hubo un aumento lineal en la evapotranspiración real del cultivo (ETr) con cantidades crecientes de riego $\left(\mathrm{R}^{2} \geq 0.97\right)$ (Djaman et al., 2013). En un suelo arenoso y condiciones secas, el riego aumentó los rendimientos y fue el factor más importante comparado con la labranza y la rotación (Huynh et al., 2019). 


\section{Dosis de nitrógeno}

La respuesta de $\mathrm{RG}$ a las dosis de $\mathrm{N}$ fue mayor a $500 \mathrm{~kg} \mathrm{~N} \mathrm{ha}^{-1}\left(p<0.01, \mathrm{R}^{2}=0.73, \mathrm{n}=51\right)$ y de $400-500 \mathrm{~kg} \mathrm{~N} \mathrm{ha}^{-1}\left(p<0.01, \mathrm{R}^{2}=0.90, \mathrm{n}=31\right.$ ). El rendimiento de grano con una propuesta de manejo varió de 16.1-19.1 $\mathrm{Mg} \mathrm{ha}^{-1}$ durante cuatro años para la meseta Loess de China y alcanzó de 98 a $108 \%$ de rendimiento potencial; la dosis optima de $\mathrm{N}$ varió de 207 a $222 \mathrm{~kg} \mathrm{ha}^{-1}$ con una proporción de 65 a $80 \mathrm{~kg}$ de grano por $\mathrm{kg}$ de $\mathrm{N}$ aplicado (Guo et al., 2016). Cuando la aplicación de $\mathrm{N}$ aumentó de 0 a $60 \mathrm{~kg} \mathrm{ha}^{-1}$, el rendimiento aumentó 7.3\% (Limón-Ortega et al., 2016).

\section{Régimen hídrico y dosis de nitrógeno}

Con esta combinación se tuvo mayor respuesta en el RG para riego $+400-500 \mathrm{~kg} \mathrm{~N} \mathrm{ha}^{-1}(p<0.01$, $\left.\mathrm{R}^{2}=0.92, \mathrm{n}=23\right)$ y riego+dosis mayor a $500 \mathrm{~kg} \mathrm{~N} \mathrm{ha}^{-1}\left(p<0.01, \mathrm{R}^{2}=0.58, \mathrm{n}=42\right)$. El rendimiento de grano con un sistema de manguera Lay Flat ${ }^{\circledR}$ fue de $10500 \mathrm{~kg} \mathrm{ha}^{-1}$, con una extracción de $\mathrm{N}$ por el grano de $165 \mathrm{~kg} \mathrm{ha}^{-1} \mathrm{y}$ una extracción de $\mathrm{N}$ por la materia seca de $109 \mathrm{~kg} \mathrm{ha}^{-1}$ (Sifuentes-Ibarra et al., 2015).

\section{Dosis de fósforo}

La mayor respuesta en el RG a través de los años al $\mathrm{P}$ aplicado fue para $275-325 \mathrm{~kg} \mathrm{P} \mathrm{ha}^{-1}(p<$ $\left.0.01, \mathrm{R}^{2}=0.45, \mathrm{n}=23\right)$ y $150-199 \mathrm{~kg} \mathrm{P} \mathrm{ha}^{-1}\left(p<0.01, \mathrm{R}^{2}=0.84, \mathrm{n}=78\right)$. No hubo respuesta en los rendimientos más allá de una dosis de $22 \mathrm{~kg} \mathrm{P} \mathrm{ha}^{-1}$, en cantidades iguales de $\mathrm{P}$ aplicado, la ausencia de una diferencia de rendimiento entre los métodos de aplicación (en banda y al voleo) indicó que la colocación en banda de $22 \mathrm{~kg} \mathrm{P} \mathrm{ha}^{-1}$ sería el mejor método de aplicación de $\mathrm{P}$ para mantener la producción del cultivo a largo plazo, bajo las prácticas de labranza manual utilizadas en la región de estudio (Smyth y Cravo, 1990).

\section{Régimen hídrico y dosis de fósforo}

$\mathrm{Al}$ combinar estos dos factores, se observó mayor respuesta en el RG para riego $+150-199 \mathrm{~kg} \mathrm{P}$ $\mathrm{ha}^{-1}\left(p<0.05, \mathrm{R}^{2}=0.62, \mathrm{n}=59\right)$ y riego+100-149 $\mathrm{kg} \mathrm{P} \mathrm{ha}^{-1}\left(p<0.01, \mathrm{R}^{2}=0.77, \mathrm{n}=30\right)$.

\section{Dosis de potasio}

La respuesta del RG fue mayor en $250-300 \mathrm{~kg} \mathrm{~K} \mathrm{ha}^{-1}\left(p<0.01, \mathrm{R}^{2}=0.92, \mathrm{n}=34\right)$ y en $145-199 \mathrm{~kg} \mathrm{~K}$ $\mathrm{ha}^{-1}\left(p<0.01, \mathrm{R}^{2}=0.72, \mathrm{n}=57\right)$. Los efectos del cloruro de $\mathrm{K}(\mathrm{KCl})$ en el verano de 2005 en dos lugares diferentes de Sulaimani: Kanypanka y Bazyan, (dosis de 0, 75, 150, 225, $300 \mathrm{~kg} \mathrm{~K} \mathrm{ha}^{-1}$ ) fueron que el aumento de la aplicación de $\mathrm{KCl}$ aumentó el rendimiento de grano en $30.17 \%$ y $55.45 \%$ para Kanypanka y Bazyan, respectivamente; la respuesta al fertilizante K se incrementó de 8.27 a $41.56 \%$ en Kanypanka, y en Bazyan fue de 3 a 34.25\% y la eficiencia del uso de fertilizante varió de 61.63 a 85.53\% para Kanypanka y de 26.6 a 54.83\% para Bazyan (Mam-Rasul, 2010).

\section{Régimen hídrico y dosis de potasio}

En esta combinación, se observó una mayor respuesta en el RG para riego+145-199 kg K ha ${ }^{-1}$ ( $p<$ $\left.0.01, \mathrm{R}^{2}=0.73, \mathrm{n}=19\right)$ y riego+250-300 $\mathrm{kg} \mathrm{K} \mathrm{ha}^{-1}\left(p<0.01, \mathrm{R}^{2}=0.94, \mathrm{n}=20\right)$. En maíz de secano, el rendimiento se redujo hasta un $13 \%$ en ausencia de K en un año (Subedi y Ma, 2009). En estrés 
por sequía, la aplicación de K en comparación con el control causó el aumento del rendimiento de grano, el peso de 1000 granos y el número de granos por mazorca en 16.5, 9.0 y 5.5\%, respectivamente (Zare et al., 2014). Las parcelas tratadas con K tuvieron un mejor rendimiento que el control en términos de mejor crecimiento, mayor rendimiento y mejoría en los componentes del rendimiento; al aumentar la dosis de $\mathrm{K}$ aplicado hasta $90 \mathrm{~kg} \mathrm{de} \mathrm{K} \mathrm{ha}^{-1}$ en dos fracciones iguales, mejoró el crecimiento y la productividad del maíz en climas semiáridos (Amanullah et al., 2016).

\section{Densidad de población}

La respuesta de $\mathrm{RG}$ aumento para densidades mayores a 7.5 plantas $\mathrm{m}^{-2}\left(p<0.01, \mathrm{R}^{2}=0.2, \mathrm{n}=74\right)$ y para 5.5-7.5 plantas $\mathrm{m}^{-2}\left(p<0.01, \mathrm{R}^{2}=0.29, \mathrm{n}=126\right)$. En la rotación maíz-soya, el aumento de la población redujo la brecha de rendimiento (tecnología alta vs tecnología estándar-TE) cuando los insumos se aplicaron a nivel suplementario a TE (Ruffo et al., 2015). El aumento de la densidad tuvo un efecto negativo en la biomasa del cultivo de cobertura, en dos sitios, el rendimiento en baja densidad (3.71 plantas $\mathrm{m}^{-2}$ ) no difirió de la estándar (7.41 plantas $\mathrm{m}^{-2}$ ) (Youngerman et al., 2018).

\section{Régimen hídrico y densidad de población}

La mayor respuesta en el RG se obtuvo en riego+densidades mayores a 7.5 plantas $\mathrm{m}^{-2}(p<0.01$, $\left.\mathrm{R}^{2}=0.21, \mathrm{n}=47\right)$ y riego+5.5-7.5 plantas $\mathrm{m}^{-2}\left(p<0.01, \mathrm{R}^{2}=0.11, \mathrm{n}=101\right)$. En condiciones de secano, el rendimiento se redujo en 8-13\% con baja densidad de plantas (PPD) (60 000 plantas ha $^{-}$ ${ }^{1}$ ) en todos los años, y el aumento de PPD a 90000 plantas ha ${ }^{-1}$ no mejoró el rendimiento (Subedi y Ma, 2009). En ambientes áridos, el rendimiento en todas las densidades fue bajo (2 $\left.448 \mathrm{~kg} \mathrm{ha}^{-1}\right)$, la variación en el rendimiento fue alta en ambientes semiáridos, la regresión polinómica $(p<0.001$, $\mathrm{n}=951$ ) tuvo su punto máximo con 140000 plantas ha $^{-1}$ y rendimiento de $9000 \mathrm{~kg} \mathrm{ha}^{-1}$, en ambientes subhúmedos el rendimiento tuvo respuesta positiva a la densidad, el rendimiento aumentó tanto para sistemas de labranza convencional y labranza cero a medida que aumentó la población, en sistemas de alto aporte de $\mathrm{N}\left(\mathrm{R}^{2}=0.19, p<0.001, \mathrm{n}=2018\right)$ la respuesta de la población al $\mathrm{N}$ aplicado fue más débil que en sistemas de aporte medio $\left(\mathrm{R}^{2}=0.49, p<0.001, \mathrm{n}=\right.$ 680) (Haarhoff et al., 2018).

\section{Cultivar}

Los materiales utilizados en los estudios fueron híbridos de empresas productoras de semillas y cruzas probadas por centros de investigación, la generalidad de los datos señaló la predominancia de los maíces amarillos, sobre los de tipo dulce y blanco, debido a datos faltantes no se realizó análisis alguno; sin embargo, se sabe que la introducción de nuevos cultivares contribuye significativamente al aumento del rendimiento del maíz (Qian y Zhao, 2017).

\section{Sistema de labranza}

Hubo respuesta a través del tiempo y una tendencia de tipo alcista en el RG obtenido en 'labranza convencional ( $\left.p<0.01, \mathrm{R}^{2}=0.69, \mathrm{n}=397\right)$ y labranza cero $\left(p<0.01, \mathrm{R}^{2}=0.56, \mathrm{n}=14\right)$. Huynh et al . (2019), encontraron una influencia negativa de la labranza cero que se hizo notar después de 3 años, lo que llevó a un rendimiento significativamente menor en comparación con la labranza convencional; sin embargo, los autores señalan que los resultados probablemente se deban a las características específicas del suelo y del sitio en donde se llevó a cabo su estudio. 


\section{Régimen hídrico y sistema de labranza}

En esta interacción hubo una respuesta significativa a través del tiempo y una tendencia alcista en el RG obtenido en riego+labranza convencional $\left(p<0.01, \mathrm{R}^{2}=0.7, \mathrm{n}=164\right)$ y secano+labranza convencional ( $\left.p<0.01, \mathrm{R}^{2}=0.87, \mathrm{n}=11\right)$. La pérdida de $\mathrm{N}$ por escorrentía y filtración, la desnitrificación causada por mayor contenido de agua del suelo y una baja tasa de mineralización de $\mathrm{N}$ causada por temperaturas bajas del suelo bajo labranza cero contribuyeron potencialmente a la disminución observada del rendimiento de grano bajo este sistema (Anapalli et al., 2018).

\section{Sistema de riego}

$\mathrm{Al}$ analizar la respuesta en el RG al sistema empleado se encontró que el riego por goteo $(p<0.01$, $\mathrm{R}^{2}=0.74, \mathrm{n}=57$ ) fue superior a los demás sistemas a través del tiempo.

\section{Sistema de cultivo}

Hubo mayor respuesta en el RG para monocultivo ( $\left.p<0.01, \mathrm{R}^{2}=0.50, \mathrm{n}=92\right)$ y rotación $(p<0.01$, $\left.\mathrm{R}^{2}=0.73, \mathrm{n}=157\right)$. La rotación más favorable del maíz fue con trigo de invierno, con adición de NPK de 60-120, 60-70 y 90-110 kg ha ${ }^{-1}$ respectivamente y densidad de 75 000-90 000 plantas $\mathrm{ha}^{-1}$, los rendimientos variaron de 2 a $11 \mathrm{t} \mathrm{ha}^{-1}$ en sistemas extensivos y de 10 a $15 \mathrm{t} \mathrm{ha}^{-1}$ en los intensivos, respectivamente (Sárvári y Pepó 2014). La rotación de cultivos aumenta y mantiene significativamente el rendimiento tanto como lo hacen el empleo de la labranza convencional y el riego (Huynh et al., 2019).

\section{Régimen hídrico y sistema de cultivo}

El efecto en el RG fue una mayor para riego + monocultivo $\left(p<0.01, \mathrm{R}^{2}=0.51, \mathrm{n}=68\right)$ y riego + rotación $\left(p<0.01, \mathrm{R}^{2}=0.81, \mathrm{n}=30\right)$.

\section{Suelo}

De acuerdo a lo reportado en los estudios consultados, el RG más alto se alcanzó en suelos con textura franco arenosa $\left(p<0.01, \mathrm{R}^{2}=0.62, \mathrm{n}=73\right)$ y franca $\left(p<0.01, \mathrm{R}^{2}=81, \mathrm{n}=61\right)$. El efecto de la interacción entre la calidad del suelo y el barbecho sobre el rendimiento del maíz fue negativo, esto sugirió la influencia de la calidad de la hojarasca y la inmovilización de $\mathrm{N}$ en los suelos (Braimoh y Vlek, 2006). La dosis óptima económica de fertilizante $\mathrm{N}$ (DOEN) fue mayor en el suelo Gleysol ( $\mathrm{Sg}$ ) (173 $\mathrm{kg} \mathrm{ha}^{-1}$ ) y más baja en el suelo Podzol (Sp) (123 kg ha ${ }^{-1}$ ) con un rendimiento de grano en Sp de casi un 60\% menor que el previsto en otros grupos texturales del suelo, la DOEN en suelos arcillosos y francos fue de 144 y $164 \mathrm{~kg} \mathrm{ha}^{-1}$ con un rendimiento de grano estimado de 12.7 y $12 \mathrm{Mg} \mathrm{ha}^{-1}$, respectivamente, el contenido del nitrato residual del suelo (NRS) fue mayor en suelos Sg y Sp, y el NRS estimado por la DOEN fue inferior al observado en el suelo Sp, lo que indica posibles pérdidas de $\mathrm{N}$ en este suelo (Alotaibi et al., 2018).

\section{Régimen hídrico y suelo}

Se observó que los valores mayores de RG se encontraron en riego + suelo franco arenoso ( $p<$ $\left.0.01, \mathrm{R}^{2}=0.69, \mathrm{n}=48\right)$ y riego+suelo franco $\left(p<0.01, \mathrm{R}^{2}=0.96, \mathrm{n}=13\right)$. 


\section{Modelos propuestos}

Las variables utilizadas en la generación del modelo fueron: lámina de riego, dosis de nitrógeno, dosis de fósforo, dosis de potasio y densidad de población, con las cuales se generaron cinco propuestas de modelos mediante método de regresión múltiple stepwise (Cuadro 3).

Cuadro 3. Modelos obtenidos y sus parámetros para la predicción del rendimiento de maíz ( $R_{\mathrm{E}}$ ).

\begin{tabular}{clccc}
\hline No. & \multicolumn{1}{c}{ Modelo $^{\text {II }}$} & $\mathrm{n}$ & $\mathrm{EE}$ & $\mathrm{R}^{2}$ \\
\hline 1 & $\mathrm{RG}_{\mathrm{E}}=3.158205+0.693319\left(\mathrm{D}_{\mathrm{P}}\right)-0.022246(\mathrm{~K})+0.005990\left(\mathrm{~L}_{\mathrm{R}}\right)+$ & 45 & 0.964 & 0.73 \\
& $0.010687(\mathrm{~N})+0.013794(\mathrm{P})$ & & & \\
2 & $\mathrm{RG}_{\mathrm{E}}=8.4373743+0.0095280\left(\mathrm{~L}_{\mathrm{R}}\right)-0.0166342(\mathrm{~K})+0.0226333(\mathrm{P})$ & 70 & 1.341 & 0.88 \\
3 & $\mathrm{RG}_{\mathrm{E}}=7.9784939+0.0085484\left(\mathrm{~L}_{\mathrm{R}}\right)+0.0084006(\mathrm{P})$ & 97 & 1.708 & 0.75 \\
4 & $\mathrm{RG}_{\mathrm{E}}=3.798942+1.082989\left(\mathrm{D}_{\mathrm{P}}\right)-0.002505(\mathrm{~K})$ & 187 & 1.742 & 0.63 \\
5 & $\mathrm{RG}_{\mathrm{E}}=8.3668051+0.5422341\left(\mathrm{D}_{\mathrm{P}}\right)-0.0071137(\mathrm{~N})+0.0026075\left(\mathrm{~L}_{\mathrm{R}}\right)$ & 81 & 1.624 & 0.35 \\
\hline
\end{tabular}

$\mathrm{RG}_{\mathrm{E}}=$ rendimiento estimado de grano; $\mathrm{n}=$ número de observaciones; $\mathrm{EE}=$ error estándar; $\mathrm{D}_{\mathrm{P}}=$ densidad de población (plantas $\mathrm{m}-2) ; \mathrm{K}=$ dosis de potasio $\left(\mathrm{kg} \mathrm{ha}^{-1}\right) ; \mathrm{L}_{\mathrm{R}}=$ lámina de riego $(\mathrm{mm}) ; \mathrm{P}=$ dosis de fósforo $\left(\mathrm{kg} \mathrm{ha}^{-1}\right) ; \mathrm{N}=$ dosis de nitrógeno $\left(\mathrm{kg} \mathrm{ha}^{-1}\right) ;{ }^{\mathrm{I}}=$ valor de $p<0.01$.

De acuerdo, al primer análisis al conjuntar las cinco variables identificadas de los estudios, el cual señaló a la densidad de población como la variable más significativa en el procedimiento stepwise, las combinaciones giraron en torno a esta variable, y se consideró como demandante de insumos (agua y fertilizantes), se eligió al modelo 1 para el reporte de resultados y su discusión debido a que consideró las cinco variables del estudio y a que presentó el menor valor del error estándar.

\section{Modelo 1}

$\mathrm{RG}_{\mathrm{E}}$ es función de las cinco variables identificadas y estudiadas en la presente investigación, los parámetros estadísticos del modelo $\left(p<0.01, \mathrm{R}^{2}=73, \mathrm{n}=45\right)$ indicaron un error de $964 \mathrm{~kg}$ grano ha $^{-1}$; en la Figura 3 se relación los $R G$ y $R_{E}$ mediante una regresión lineal, cuya ecuación fue altamente significativa ( $p<0.01, \mathrm{R}^{2}=73$ ); asimismo, se observó la tendencia a la subestimación de los valores por parte del modelo generado.

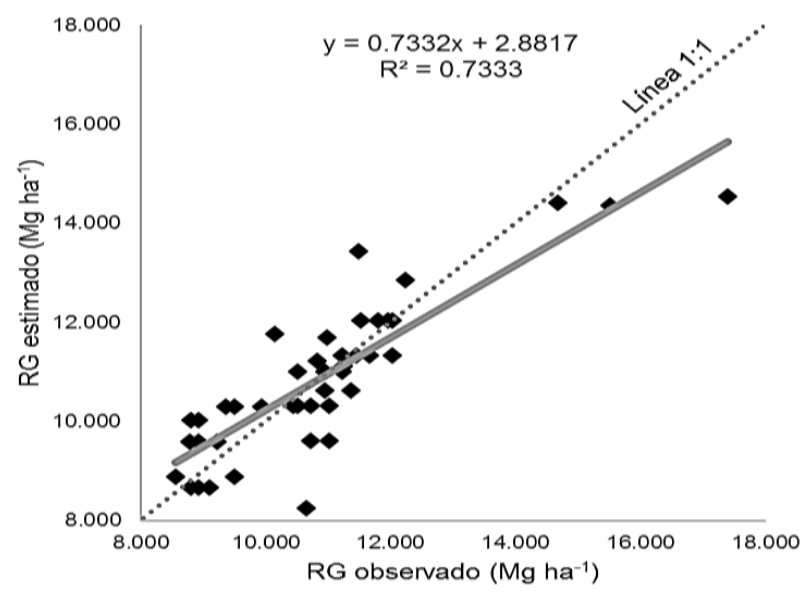

Figura 3. Relación entre los datos observados y los estimados con el modelo 1. La línea punteada 3 indica la relación 1:1 entre el valor observado $(R G)$ y el estimado $\left(R_{\mathrm{E}}\right)$. 
Al respecto, De la Casa (1992) halló una ecuación de regresión múltiple $\left(\mathrm{R}^{2}=0.8, \mathrm{EE}=407.1 \mathrm{~kg}\right.$ $\mathrm{ha}^{-1}$ ), basada en datos de precipitación, al utilizar el método stepwise para predecir el rendimiento de maíz en Argentina, constó de tres componentes, uno tecnológico o temporal, uno pluviométrico, y uno geográfico, dicha ecuación alcanzó un error porcentual medio entre 17 y $18 \%$ respecto de los datos testigos. Monteiro et al. (2017) estimaron mediante un modelo el rendimiento del maíz para de la temporada 2000-2001 a la 2007-2008 en Brasil basado en el nivel tecnológico de los sistemas de producción y las condiciones meteorológicas, hubo una alta correlación entre el rendimiento estimado y el observado $\left(0.76>\mathrm{R}^{2} \geq 0.92, p<0.01\right)$ con la eficiencia del modelo entre 0.45 y 0.73 , error medio relativo (MAE) entre -0.9 y 2.4 , y error medio absoluto (MAE) inferior a $70 \mathrm{~kg} \mathrm{ha}^{-1}$ dependiendo del nivel tecnológico.

\section{Conclusiones}

Los factores que influyeron en la obtención del $\mathrm{RG}_{\mathrm{E}}$ en maíz fueron: densidad de población, dosis de $\mathrm{K}$, lámina de riego, dosis de $\mathrm{N}$ y dosis de $\mathrm{P}$ y todos ellos pertenecieron al grupo de factores de tipo agronómico. De acuerdo, al análisis de estudios relacionados a la obtención del rendimiento de grano de maíz y a la diversidad de ambientes en donde se desarrollaron, la influencia de factores agronómicos se asoció de manera significativa con la respuesta del cultivo, por lo cual sólo se eligieron estas variables para determinar el $\mathrm{RG}_{\mathrm{E}}$. El modelo de regresión $\left(n=45, \mathrm{R}^{2}=0.73\right.$, EE.= $0.964 \mathrm{Mg} \mathrm{ha}^{-1}$ ) representó el efecto de factores de tipo agronómico sobre el RG, mientras que las variables ambientales no repercutieron de manera significativa sobre la respuesta del cultivo.

\section{Literatura citada}

Alcántar-González, G.; Trejo-Téllez, L. I. y Gómez-Merino, F. C. 2016. Nutrición de cultivos. 2ª . (Ed.). Biblioteca Básica de Agricultura, México, DF. 443 p.

Alotaibi, K. D.; Cambouris, A. N.; Luce, M. S.; Ziadi, N. and Tremblay, N. 2018. Economic optimum nitrogen fertilizer rate and residual soil nitrate as influenced by soil texture in corn production. Agron. J. 110(6):2233-2242.

Amanullah, I. A.; Irfanullah. and Hidayat, Z. 2016. Potassium management for improving growth and grain yield of maize (Zea mays L.) under moisture stress condition. Sci. Rep. 6:1-12.

Anapalli, S. S.; Reddy, K. N. and Jagadamma, S. 2018. Conservation tillage impacts and adaptations in irrigated corn production in a humid climate. Agron. J. 110(6):2673-2686.

Assefa, Y.; Vara-Prasad, P. V.; Carter, P.; Hinds, M.; Bhalla, G.; Schon, R.; Jeschke, M.; Paszkiewicz, S. and Ciampitti, I. A. 2017. A new insight into corn yield: trends from 1987 through 2015. Crop Sci. 57(5):2799-2811.

Braimoh, A. K. and Vlek, P. L. G. 2006. Soil quality and other factors influencing maize yield in northern Ghana. Soil Use and Management. 22(2):165-171.

Bugarín-Montoya, R.; Galvis-Spinola, A.; Sánchez-García, P. y García-Paredes, D. 2002. Acumulación diaria de materia seca y de potasio en la biomasa aérea total del tomate. Terra. 20(4):401-409.

Ciampitti, I. A.; Boxler, M. y García, F. O. 2010. Nutrición de maíz: requerimientos y absorción de nutrientes. Informaciones Agronómicas del Cono Sur. 48:14-18.

De la Casa, A. 1992. Modelo estadístico de pronóstico de rendimiento de maíz para la región semiárida de Córdoba basado en datos pluviométricos areales. Agriscientia. 9(2):87-96. 
Djaman, K.; Irmak, S.; Rathje, W. R.; Martin, D. L. and Eisenhauer, D. E. 2013. Maize evapotranspiration, yield production functions, biomass, grain yield, harvest index, and yield response factors under full and limited irrigation. Am. Society Agric. Biol. Eng. 56(2):273-293.

García, F. 2005. Criterios para el manejo de la fertilización del cultivo de maíz. In: La jornada "Maíz 2005" organizada por Capacitación Agropecuaria. Córdoba, Argentina. 1-19 pp.

García, J. M. 2008. Teoría y ejercicios prácticos de dinámica de sistemas. Fundación Universitat Politécnica de Catalunya (UPC). Cataluña, España. 84 p.

Greenwood, D. J. 1983. Quantitative theory and the control of soil fertility. New Phytol. 94(1):1-18.

Guo, J.; Wang, Y.; Fan, T.; Chen, X. and Cui, Z. 2016. Designing corn management strategies for high yield and high nitrogen use efficiency. Agron. J. 108(2):922-929.

Haarhoff, S. and Swanepoel, P. A. 2018. Plant population and maize grain yield: a global systematic review of rainfed trials. Crop Sci. 58(5):1819-1829.

Huynh, H. T.; Hufnagel, J.; Wurbsa, A. and Bellingrath-Kimura, S. D. 2019. Influences of soil tillage, irrigation and crop rotation on maize biomass yield in a 9-year field study in Müncheberg, Germany. Field Crops Res. 241:1-9.

Jun, X.; Rui-Zhi, X.; Wang-Feng, Z.; Ke-Ru, W.; Peng, H.; Bo, M.; Ling, G. and Shao-Kun, L. 2017. Research progress on reduced lodging of high-yield and -density maize. J. Integrative Agric. 16(12):2717-2725.

Kovács, P. and Vyn, T. J. 2017. Relationships between ear-leaf nutrient concentrations at silking and corn biomass and grain yields at maturity. Agron. J. 109(6):2898-2906.

Leeper, R. A.; Runge, E. C. A. and Walker, D. M. 1974. Effect of plant-available stored soil moisture on corn yields. I. Constant Climatic Conditions. Agron. J. 66(6):723-727.

Limón-Ortega, A.; Ruiz-Torres, N. A.; Vázquez-Carrillo, G. and Báez-Pérez, A. 2016. Environment and nitrogen influence on rainfed maize yield and quality. Crop Sci. 56(3):1257-1264.

Liu, B.; Chen, X.; Meng, Q.; Yang, H. and Van Wart, J. 2017. Estimating maize yield potential and yield gap with agro-climatic zones in China-Distinguish irrigated and rainfed conditions. Agric. Forest Meteorol. 239:108-117.

Mam-Rasul, G. A. 2010. Effect of potassium fertilizer on growth and yield of corn plants in some soils at Sulaimani governorate. Mesopotamia J. Agric. 38(1):35-43.

Mason, S. C.; Kent, D. K.; Eskridge, M. and Galusha, T. D. 2008. Yield increase has been more rapid for maize than for grain sorghum. Crop Sci. 48(4):1560-1568.

Melgar, R. y Torres-Duggan, M. 2004. Manejo de la fertilización en maíz. IDIA XXI. Revista de Información sobre Investigación y Desarrollo Agropecuario. 4(6):114-121.

Monteiro, J. E. B. de A.; Assad, E. D.; Sentelhas, P. C. and Azevedo, L. da C. 2017. Modeling of corn yield in Brazil as a function of meteorological conditions and technological level. Pesq. Agropec. Bras. 52(3):137-148.

Phillips, R. L. 2010. Mobilizing science to break yield barriers. Crop Sci. 50(1):99-108.

Qian, J. and Zhao, Z. 2017. Estimating the contribution of new seed cultivars to increases in crop yields: a case study for corn. Sustainability. 9(7):1-11.

R Core Team. 2017. R: a language and environment for statistical computing. https://www.rproject.org/.

Ruffo, M. L.; Gentry, L. F.; Henninger, A. S.; Seebauer, J. R. and Below, F. E. 2015. Evaluating management factor contributions to reduce corn yield gaps. Agron. J. 107(2):495-505. 
Sangoi, L. 2000. Understanding plant density effects on maize growth and development: an important issue to maximize grain yield. Ciência Rural. 31(1):159-168.

Sárvári, M. and Pepó, P. 2014. Effect of production factors on maize yield and yield stability. Cereal Res. Comm. 42(4):710-720.

Shisanya, C. A.; Mucheru, M. W.; Mugendi, D. N. and Kung'u, J. B. 2009. Effect of organic and inorganic nutrient sources on soil mineral nitrogen and maize yields in central highlands of Kenya. Soil Tillage Res. 103(2):239-246.

Sifuentes-Ibarra, E.; Macías-Cervantes, J.; Ruelas-Islas, J. R.; Preciado-Rangel, P.; OjedaBustamante, W.; Inzunza-Ibarra, M. A. y Samaniego-Gaxiola, J. A. 2015. Mejoramiento del grado de uso del nitrógeno en maíz mediante técnicas parcelarias de riego por superficie. Rev. Mex. Cienc. Agríc. 6(8):1903-1914.

Smyth, T. J. and Cravo, M. S. 1990. Phosphorus management for continuous corn-cowpea production in a Brazilian Amazon Oxisol. Agron. J. 82(2):305-309.

Subedi, K. D. and Ma, B. L. 2009. Assessment of some major yield-limiting factors on maize production in a humid temperate environment. Field Crops Res. 110(1):21-26.

Tagarakis, A. C. and Ketterings, Q. M. 2017. In-season estimation of corn yield potential using proximal sensing. Agron. J. 109(4):1323-1330.

Tao, F.; Zhang, S.; Zhang, Z. and Rötter, R. P. 2015. Temporal and spatial changes of maize yield potentials and yield gaps in the past three decades in China. Agric. Ecosystems Environ. 208:12-20.

Van Loon, M. P.; Adjei-Nsiah, S.; Descheemaeker, K.; Akotsen-Mensah, C.; Van Dijk, M.; Morley, T.; Van Ittersum, M. K. and Reidsma, P. 2019. Can yield variability be explained? Integrated assessment of maize yield gaps across smallholders in Ghana. Field Crops Res. 236:132-144.

Verdugo-Vásquez, N.; Acevedo-Opazo, C.; Valdés-Gómez, H.; Araya-Alman, M.; Ingram, B.; García de Cortázar-Atauri, I. and Tisseyre, B. 2016. Spatial variability of phenology in two irrigated grapevine cultivar growing under semi-arid conditions. Precis. Agric. 17(2):218-245.

Wang, J.; Wang, E.; Yin, H.; Feng, L. and Zhang, J. 2014. Declining yield potential and shrinking yield gaps of maize in the North China Plain. Agric. Forest Meteorol. 195-196:89-101.

Youngerman, C. Z.; DiTommaso, A.; Curran, W. S.; Mirsky, S. B. and Ryan, M. R. 2018. Corn Density Effect on Interseeded Cover Crops, Weeds, and Grain Yield. Agron. J. 110(6):2478-2487.

Zare, K.; Vazin, F. and Hassanzadehdelouei, M. 2014. Effects of potassium and iron on yield of corn (Zea mays L.) in drought stress. Cercetări Agronomice în Moldova. 47(1):39-47.

Zhao, J.; Yang, X. and Sun, S. 2018. Constraints on maize yield and yield stability in the main cropping regions in China. Eur. J. Agron. 99:106-115. 